\title{
Pyrethroid and Carbamate Resistance in Anopheles funestus Giles along Lake Kariba in Southern Zambia
}

\author{
Javan Chanda, ${ }^{1 *}$ Kochelani Saili, ${ }^{1}$ Foustina Phiri, ${ }^{2}$ Jennifer C. Stevenson, ${ }^{3,4}$ Mulenga Mwenda, ${ }^{1}$ Sandra Chishimba ${ }^{1}$ \\ Conceptor Mulube, ${ }^{1}$ Brenda Mambwe, ${ }^{1}$ Christopher Lungu, ${ }^{1}$ Duncan Earle, ${ }^{1}$ Adam Bennett, ${ }^{5}$ Thomas P. Eisele, ${ }^{6}$ \\ Mulakwa Kamuliwo, ${ }^{2}$ Richard W. Steketee, ${ }^{7}$ Joseph Keating, ${ }^{6}$ John M. Miller, ${ }^{1}$ and Chadwick H. Sikaala ${ }^{2,8}$ \\ ${ }^{1}$ PATH Malaria Control and Elimination Partnership in Africa (MACEPA), Lusaka, Zambia; ${ }^{2}$ National Malaria Elimination Centre, Zambia Ministry of \\ Health, Lusaka, Zambia; ${ }^{3}$ Macha Research Trust, Choma, Zambia; ${ }^{4}$ W. Harry Feinstone Department of Molecular Microbiology and Immunology, \\ Johns Hopkins Bloomberg School of Public Health, Baltimore, Maryland; ${ }^{5}$ Malaria Elimination Initiative, Global Health Group, University of \\ California San Francisco, San Francisco, California; ${ }^{6}$ Department of Tropical Medicine, Center for Applied Malaria Research and Evaluation, Tulane \\ University School of Public Health and Tropical Medicine, New Orleans, Louisiana; ${ }^{7}$ PATH MACEPA, Seattle, Washington; ${ }^{8}$ SADC Malaria \\ Elimination Eight Secretariat, Windhoek, Namibia
}

\begin{abstract}
Whereas data on insecticide resistance and its underlying mechanisms exist for parts of Zambia, data remain limited in the southern part of the country. This study investigated the status of insecticide resistance, metabolic mechanisms, and parasite infection in Anopheles funestus along Lake Kariba in southern Zambia. Indoor-resting mosquitoes were collected from 20 randomly selected houses within clusters where a mass drug administration trial was conducted and raised to F1 progeny. Non-blood-fed 2- to 5-day-old female An. funestus were exposed to WHO insecticide-impregnated papers with $0.05 \%$ deltamethrin, $0.1 \%$ bendiocarb, $0.25 \%$ pirimiphos-methyl, or $4 \%$ dichlorodiphenyl-trichloroethane (DDT). In separate assays, An. funestus were pre-exposed to piperonyl butoxide (PBO) to determine the presence of monooxygenases. Wild-caught $A n$. funestus that had laid eggs for susceptibility assays were screened for circumsporozoite protein of Plasmodium falciparum by ELISA, and sibling species were identified by polymerase chain reaction. Anopheles funestus showed resistance to deltamethrin and bendiocarb but remained susceptible to pirimiphos-methyl and DDT. The pre-exposure of An. funestus to PBO restored full susceptibility to deltamethrin but not to bendiocarb. The overall sporozoite infection rate in An. funestus populations was 5.8\%. Detection of pyrethroid and carbamate resistance in An. funestus calls for increased insecticide resistance monitoring to guide planning and selection of effective insecticide resistance management strategies. To prevent the development of resistance and reduce the underlying vectorial capacity of mosquitoes in areas targeted for malaria elimination, an effective integrated vector management strategy is needed.
\end{abstract}

\section{INTRODUCTION}

Over the last 15 years, long-lasting insecticidal nets (LLINs), indoor residual spraying (IRS), intermittent preventive treatment, prompt diagnosis, and treatment with artemisininbased combination therapy have been scaled-up for malaria control and elimination in sub-Saharan Africa, reducing malaria incidence by $22 \%$ and mortality by $29 \%$ between 2010 and 2018. ${ }^{1}$ Equally in Zambia, malaria incidence declined by $5 \%$ and mortality by $55 \%$ between 2010 and 2018 . $^{2}$ Most of the gains reported in malaria reduction in sub-Saharan Africa are attributed to the rapid scale-up of vector control interventions. ${ }^{3}$ However, the emergence of insecticide resistance in major malaria vectors puts these fragile gains at risk. ${ }^{4,5}$

In Zambia, Plasmodium falciparum account for $98 \%$ of all malaria cases reported at health facilities. ${ }^{2}$ Malaria transmission is maintained by three major vectors: An. gambiae sensu stricto Giles, Anopheles arabiensis Paton, and Anopheles funestus sensu stricto Giles. ${ }^{6}$ To effectively control these malaria vectors and reduce malaria transmission, the Ministry of Health $(\mathrm{MoH})$ through the National Malaria Control Programme has scaled-up LLINs and IRS for malaria control and elimination in the country. Operational vector control with LLINs and IRS in Zambia depends on five classes of insecticides recommended by the $\mathrm{WHO}^{7}$ : pyrethroids, carbamates, organophosphates, organochlorines, and neonicotinoids. Of

\footnotetext{
*Address correspondence to Javan Chanda, PATH Malaria Control and Elimination Partnership in Africa, Stand No. 11059, Mikwala House, Off Brentwood Lane, Longacres, P.O. Box 32509, Lusaka 10101, Zambia. E-mail: jchanda@path.org
}

these insecticides, pyrethroids, are the only insecticide class currently the $\mathrm{WHO}$ recommended for bednet impregnation because of their effectiveness, low toxicity to humans, and high excito-repellent effects on mosquitoes. ${ }^{8}$ A new-generation mixture LLIN called Interceptor ${ }^{\circledR}$ G2 (BASF $^{\odot}$, Ludwigshafen, Germany) that combines chlorfenapyr (a pyrrole) and alphacypermethrin (a pyrethroid) has been developed by BASF $^{\odot}$ (Ludwigshafen, Germany) and prequalifications listed by the $\mathrm{WHO}^{9}$ but has yet to be rolled out widely. Entomological studies conducted in Zambia and Tanzania have demonstrated the impact of using either LLINs or IRS on reducing malaria vector abundance, infection rates, and malaria transmission. ${ }^{10-12}$ Nonetheless, the effectiveness of LLINs and IRS depends on high coverage within the community, mosquito susceptibility to insecticides used, and indoor-biting and resting behaviors of mosquitoes.

Long-lasting insecticidal nets and IRS exploit the biting and resting behaviors of local malaria vectors to reduce malaria transmission. Long-lasting insecticidal nets are designed to reduce human-vector contact by targeting night-biting mosquitoes, whereas IRS aims to reduce the life span of indoor-biting (endophagic) and indoor-resting (endophilic) mosquitoes. ${ }^{13}$ The endophagic and endophilic behavioral characteristics of mosquitoes tend to expose them to insecticides through contact with LLINs and or IRS. ${ }^{14}$ In addition, the same insecticides used for public health are equally used in agriculture for pest control and usually end up contaminating mosquito-breeding sites. ${ }^{15}$ In this regard, there has been increased selection pressure on mosquito populations that demonstrate either physiological or behavioral resistance to different classes of insecticides. The common mechanisms 
responsible for physiological resistance are metabolic detoxification and decreased target-site sensitivity. ${ }^{16}$ In metabolic detoxification, the insecticide is prevented from reaching the site of action in lethal concentration by detoxifying enzyme groups (P450s monooxygenases, glutathione s-transferases, and esterases). ${ }^{16}$ By contrast, decreased target-site sensitivity mechanism reduces the rate at which the insecticide binds to its target site (knockdown resistance [kdr], dieldrin resistance $[r d l]$, and acetylcholinesterase [ace.1]). ${ }^{16-18}$ The other mechanisms of resistance include penetration resistance which occurs as a result of cuticle thickening ${ }^{19}$ and behavioral resistance where mosquitoes may evolve to avoid insecticidal contact in malaria vectors. ${ }^{20,21}$

Following a decade of scaling-up LLINs and IRS in Zambia, major malaria vectors An. funestus s.s. and An. gambiae s.s. developed resistance to the dichloro-diphenyl-trichloroethane (DDT) and pyrethroids. ${ }^{22,23}$ The detection of cross-resistance to pyrethroids and organochlorines in 2010 prompted the Zambian National Malaria Control Programme to change its policy, transitioning from using long-lasting DDT to short-lived carbamates and organophosphates during the 2011 and 2012 IRS campaigns. ${ }^{23}$ Countrywide entomological monitoring conducted between 2012 and 2013 revealed high resistance to carbamates and pyrethroids in An. funestus s.s. and $A n$. gambiae s.s. populations. ${ }^{24,25}$ As a result, in 2013, the National Malaria Control Programme stopped using carbamates and pyrethroids for IRS and adopted the organophosphate pirimiphos-methyl 300 CS as the Zambia's IRS insecticide of choice, using it annually from 2014 through 2018 spray season. ${ }^{23,26}$

The Zambian $\mathrm{MoH}$, in collaboration with its partners, has an ambitious goal of eliminating malaria nationally by 2021 . To eliminate malaria in southern Zambia, mass distribution of LLINs and annual deployment of IRS are being scaled-up alongside expanded case management and mass drug administration (MDA) strategies. Mass drug administration with dihydroartemisinin-piperaquine (DHAp) is implemented at the community scale to clear $P$. falciparum infections and reduce human parasite reservoirs in Southern Province. ${ }^{27}$ To accelerate malaria elimination in low-transmission areas of Southern Province, MDA is effective when implemented in areas with good access to case management, strong surveillance, and universal coverage of vector control. ${ }^{27}$ Anopheles funestus is one of the major vectors of human malaria in much of Zambia, including parts of Southern Province. ${ }^{28}$ High parasite rates ranging from $6 \%$ to $20.0 \%$ have been reported in An. funestus from six provinces of Zambia. ${ }^{25}$ To reduce the vectorial capacity of An. funestus in the Southern Province of Zambia, the coverage of LLINs and IRS has increased at health facility catchment areas. With increased coverage of insecticide-based interventions, populations of An. funestus have developed resistance to pyrethroids, carbamates, and organochlorines in Zambia, ${ }^{22,29-31}$ as reported in almost every sub-Saharan African country. ${ }^{16,30}$ The resistance profiles observed in An. funestus from Zambia relate to the upregulation of cytochrome P450 oxidases. ${ }^{22,25,29,31}$ Presently, there is no case of kdr target-site mutations that has been detected in An. funestus populations in Zambia or elsewhere. ${ }^{32}$ However, data on the impact of universal coverage of vector control on insecticide resistance and its mechanisms in the malaria vectors along Lake Kariba of southern Zambia are limited. To ensure that insecticide choice is effective and sustainable for malaria vector control programs, the Global Plan for Insecticide Resistance Management encourages continuous monitoring of insecticide resistance and identification of resistance mechanisms in malaria major vector populations. $^{33}$

In the context of locally aggressive malaria control and elimination, this study provides updated information on $A n$. funestus susceptibility status to the four classes of insecticides, underlying metabolic resistance mechanisms, and $P$. falciparum sporozoite infection rates in Southern Province, Zambia. The results of this study also provide empirical evidence required to guide policy formulations and strategic implementation of sustainable insecticide resistance management strategies aimed at reducing malaria transmission in endemic areas of Southern Province.

\section{MATERIALS AND METHODS}

Study areas. The study was conducted in six study sites where an MDA trial was being implemented in Southern Province, Zambia (Figure 1). Details of the trial setup are given elsewhere. ${ }^{27}$ Entomological collections of mosquitoes in the study sites were performed from April 2015 to May 2015. The study sites were distributed along Lake Kariba in two districts, Siavonga and Sinazongwe. Mass distribution of pyrethroidonly LLINs, using PermaNet ${ }^{\circledR} 2.0$ (Vestergaard Frandsen, Saint Francois, Switzerland) and Olyset Net ${ }^{\circledR}$ (Sumitomo Chemicals, Chuo, Japan), and IRS with the organophosphate pirimiphosmethyl (Actellic ${ }^{\circledR} 300$ SC, Syngenta, Basel, Switzerland) were the primary vector control tools used in the study areas. Scaled malaria case management by community health workers was supplemented with reactive case detection to areas surrounding index case households to identify additional infections. ${ }^{34}$ In addition, as part of the MDA trial, targeted MDA with DHAp was being conducted to clear malaria parasites from the local human populations in the study areas of Southern Province. ${ }^{27}$

The rainfall pattern in Southern Province is seasonal with the main rainy season starting in December and ending in April. Previous studies have shown that Anopheles arabiensis and An. funestus s.s. are the principal malaria vector species in Southern Province. $22,35,36$ The main economic activities practiced in the study areas of Southern Province are fishing and agriculture. To protect animals and crops from pests, farmers use different classes of insecticides in Southern Province as shown in Supplemental Table S1.

Mosquito collections and rearing. Indoor-resting bloodfed and gravid female Anopheles mosquitoes were collected in 20 randomly selected houses per site using a CDC Backpack Aspirator (John W. Hock Company) between 4:00 and 5:00 AM. The collected adult mosquitoes were kept in BugDorm cages (Mega Science co. Ltd, Taichung, Taiwan) covered with moist cotton towels to support survival. The mosquitoes were later transported to the central laboratory at the National Malaria Control Centre in Lusaka for further processing. Gravid mosquitoes were induced to lay eggs in perforated Eppendorf tubes according to the protocol described by Morgan et al. ${ }^{37}$ After laying eggs, adult mosquitoes were removed and stored in well-labeled Eppendorf tubes. The eggs on the filter paper were immersed in paper cups filled with mineral water (Manzivalley ${ }^{\circledR}$ Ltd, Natural Valley Limited, Chongwe, Zambia) for the development of aquatic mosquito stages. Rearing of mosquito larvae and pupae to F1s was 


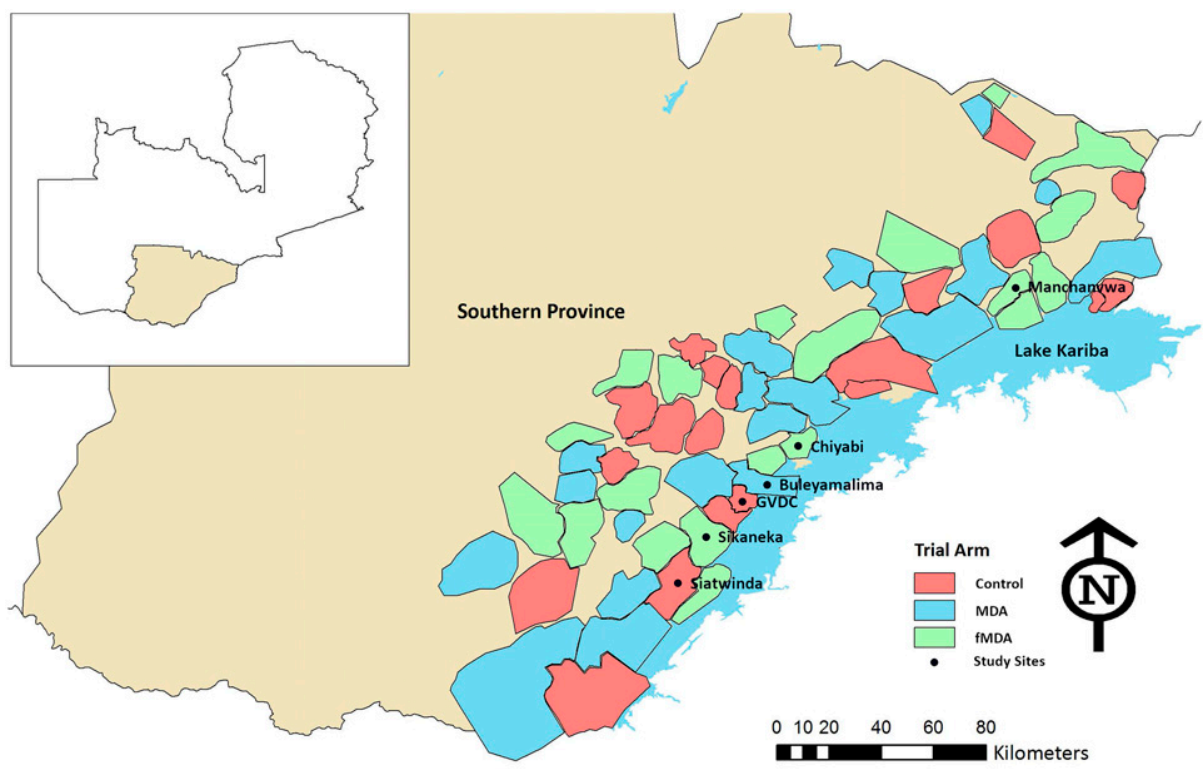

FIGURE 1. Map of Southern Province showing six study areas in Sinazongwe and Siavonga districts of Zambia, 2015.

conducted under climate-controlled standard laboratory conditions with a temperature range of $26 \pm 2^{\circ} \mathrm{C}$ and $70-80 \%$ relative humidity.

WHO susceptibility tests. Bioassays were conducted using WHO tube kits to assess susceptibility or resistance of the F1 adult mosquitoes that emerged from those caught from the study sites. Four insecticide-impregnated papers were used: organochlorine (4\% DDT), pyrethroid (0.05\% deltamethrin), carbamate $(0.1 \%$ bendiocarb), and organophosphate $(0.25 \%$ pirimiphos-methyl), all procured from a WHO-collaborating center in Malaysia. Four batches of 20-25 unfed An. funestus females aged 2-5 days were exposed to each set of insecticidetreated papers for 60 minutes and maintained at $26 \pm 2{ }^{\circ} \mathrm{C}$ and $70-80 \%$ relative humidity in the insectary. The knockdown effects on An. funestus were monitored at 10-minute intervals over 60 minutes of exposure time. Final mortality of $A n$. funestus was scored after a 24-hour post-exposure period, during which survivors were supplied with a $10 \%(\mathrm{w} / \mathrm{v})$ sugar solution according to the $\mathrm{WHO}$ standard procedure. ${ }^{38}$ A negative control where wild-caught female An. funestus were exposed to untreated papers in the tube assays was used each time of testing. In the absence of a susceptible An. funestus strain for a positive control at the central laboratory, the susceptible colony of $A n$. gambiae s.s. Kisumu strain was used to check the quality of insecticide-impregnated papers during bioassays.

Piperonyl butoxide (PBO) synergist tests. To understand the role of metabolic resistance in the $A n$. funestus population, PBO, a synergist, was used in this study. Piperonyl butoxide inhibits the specific activity of P450 monooxygenases in insect populations. ${ }^{39}$ Subsamples of 20-25 unfed, 2- to 5day-old F1 generation An. funestus were pre-exposed to $4 \%$ PBO-impregnated test papers for one hour. After pre-exposure to $\mathrm{PBO}, \mathrm{An}$. funestus populations were immediately exposed to $0.05 \%$ deltamethrin or $0.1 \%$ bendiocarb for an additional hour. ${ }^{40}$ One batch of 20-25 An. funestus were only exposed to $4 \%$ PBO without insecticide to serve as a control. After pre-exposure to PBO and either of the insecticides, An. funestus mosquitoes were transferred back to holding tubes and supplied with $10 \%$ sugar solution. Final mortality of both the controls and the An. funestus exposed to insecticides were scored after 24 hours.

Parasite infection. Sandwich ELISA was conducted on the head and thorax segments of dried random samples of wild-caught An. funestus that had laid eggs for resistance susceptibility bioassays. The detection of $P$. falciparum in populations of An. funestus was analyzed according to the protocol of Wirtz et al. ${ }^{41}$

Species identification. All field-collected mosquitoes were morphologically identified using the standard mosquito identification keys described by Gillies and De Meillon ${ }^{42}$ and Gillies and Coetzee, ${ }^{43}$ and DNA was extracted from morphologically identified $A n$. funestus mosquitoes from either the wings or legs of each mosquito sample. ${ }^{44}$ Sibling species of the An. funestus group were identified using a multiplex polymerase chain reaction (PCR) assay, and the PCR products were visualized using gel electrophoresis. ${ }^{45}$

Data analysis. Data were analyzed using Microsoft Excel ${ }^{\circledR}$ (Microsoft Corporation, Redmond, WA) software. The prevalence of insecticide resistance in An. funestus was defined as per the standard $\mathrm{WHO}$ protocol by calculating mortality rate percentage 24 hours post-exposure. Effects of PBO on the mortality of An. funestus populations were determined by Student's paired $t$-test. Binary logistic regression analysis using the generalized estimating equation (GEE) model accounting for clustering by using the replicates or batches of mosquitoes exposed in each WHO tube as the repeated measure was used to determine the difference in levels of susceptibility in An. funestus populations to insecticides between study sites. In this study, a $P$-value of $\leq 0.05$ was considered to be significant.

Ethical consideration. Before mosquito collections, village meetings with community leaders and households were 
conducted to seek permission to trap mosquitoes in the study areas of Southern Province, Zambia.

\section{RESULTS}

Mosquito species composition. A total of 2,516 indoor, blood-fed, and gravid mosquitoes were collected in 20 randomly selected houses in each study area using a CDC Backpack Aspirator, of which 76\% $(n=1,912)$ were morphologically identified as An. funestus s.I. Giles, $16 \%(n=402)$ as An. gambiae s.l. Giles, and the remaining $8 \%(n=202)$ comprised other anophelines: An. coustani s.l. Laveran, An. squamosus Theobald, An. rufipes Gough, and An. pretoriensis Theobald.

Species identification. Polymerase chain reaction was performed on a random subsample $(n=310)$ of An. funestus that had laid eggs for insecticide resistance bioassays. Polymerase chain reaction results confirmed that $82.2 \%(n=255)$ were An. funestus s.s., $11.6 \%(n=36)$ An. rivulorum, and 19 samples failed to amplify as summarized in Figure 2.

Insecticide susceptibility tests. Results of WHO bioassays carried out on F1 generation An. funestus are summarized in Table 1. The mortality rate of $A n$. gambiae s.s. Kisumu-susceptible laboratory-reared strain exposed to treated papers serving as a positive control was 100\% $(n=$ 200) for all tested insecticides. For the negative control, mortality rates of $A n$. funestus were below $5 \%$ (Table 1). Based on the WHO criteria of $2013,,^{38}$ mortality rates of An. funestus confirmed resistance to two insecticides, deltamethrin, and bendiocarb. Resistance to $0.05 \%$ deltamethrin (pyrethroid) was detected in An. funestus with mortality rates at 24 hours ranging from $13.8 \%$ (95\% Cl: 6.8-20.7) in the Gwembe Valley Development Centre (GVDC) to 52.0\% (95\% Cl: 38.1-65.8) in Manchanvwa (Table 1). Exposure of An. funestus to $0.1 \%$ bendiocarb (carbamate) showed resistance with mortality rates at 24 hours ranging from $40.9 \%$ (95\% Cl: 30.9-50.8) in GVDC to $66.7 \%$ (95\% Cl: 55.3-78.0) in Manchanvwa(Table 1). Based on the estimates of the deltamethrin and bendiocarb models, there was a significant difference in the susceptibility of $A n$. funestus to deltamethrin between study sites (GEE, $P<$ $0.05)$, but no difference in study sites was observed with bendiocarb. By contrast, all populations of $A n$. funestus were susceptible to $0.25 \%$ pirimiphos-methyl (organophosphate) and 4\% DDT (organochlorine), with 100\% mortality recorded at 24 hours post-exposure period across the study areas.

Piperonyl butoxide bioassays. Tables 2 and 3 show the role of metabolic resistance in populations of An. funestus using a synergist PBO. Pre-exposure of pyrethroid-resistant An. funestus to PBO increased the mortality rates to $100 \%$ against deltamethrin across the study sites (Table 2). The effects of PBO on pyrethroid-resistant An. funestus population were statistically significant (Table 2), excluding Sikaneka (paired sample Student's $t$-test; $t=7.667$, df $=1$, and $P=$ 0.0825). By contrast, PBO restored the full susceptibility
Manchanvwa $\mathrm{n}=70$

an. funestus s.s $\square$ An. rivulorum

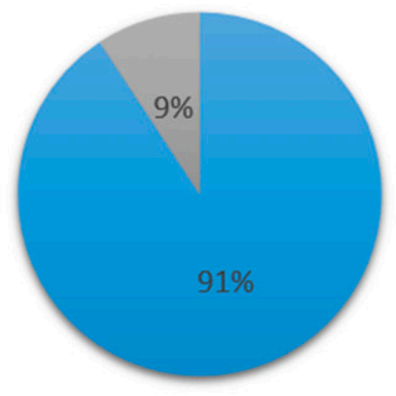

Siatwinda $n=22$

- An. funestus s.s $₫$ An. rivulorum

- Unamplified

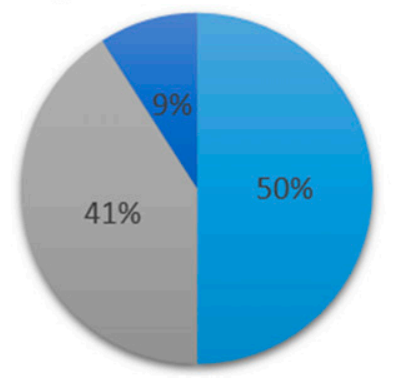

Chiyabi $n=62$

An. funestus s.s $\quad$ An. rivulorum

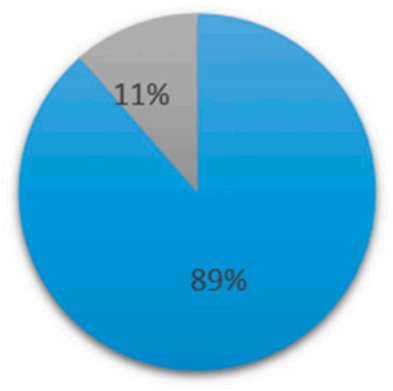

GVDC $n=83$

$\square$ An.funestus s.s $\square$ An. rivulorum

—unamplified

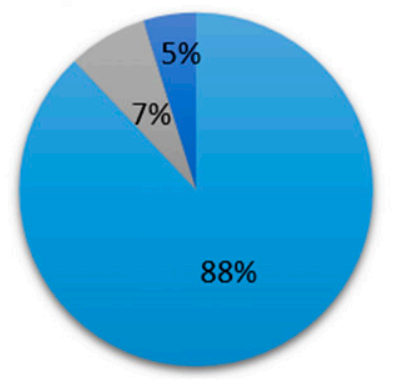

Buleyamalima $n=68$

- An. funestus s.s $\square$ An. rivulorum

घ Unamplified

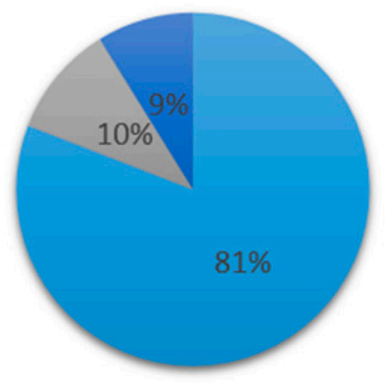

Sikaneka $n=13$

an. funestus s.s $\square$ An. rivulorum

- Unamplified

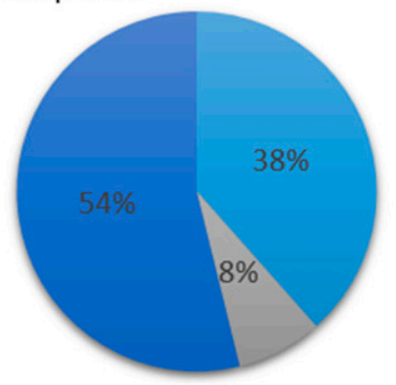

FIGURE 2. Vector species composition in six study areas of Southern Province, Zambia, 2015. 
TABLE 1

WHO susceptibility bioassay test results on 2- to 5-day-old F1 Anopheles funestus s.l. in the Southern Province of Zambia, 2015

\begin{tabular}{|c|c|c|c|c|}
\hline & Deltamethrin $(0.05 \%)$ & Bendiocarb $(0.1 \%)$ & Pirimiphos-methyl (0.25\%) & Dichloro-diphenyl-trichloroethane (4.0\%) \\
\hline Study site & $\%$ Mortality $(n)$ & $\%$ Mortality $(n)$ & $\%$ Mortality $(n)$ & $\%$ Mortality $(n)$ \\
\hline Buleyamalima & $27.0(100)$ & $55.3(94)$ & $100(44)$ & $100(54)$ \\
\hline Gwembe Valley Development Centre & $13.8(94)$ & $40.9(93)$ & $100(100)$ & $100(99)$ \\
\hline Chiyabi & $28.0(32)$ & $47.0(60)$ & $100(50)$ & $100(45)$ \\
\hline Sikaneka & $42.3(26)$ & $56.0(50)$ & nd & $100(52)$ \\
\hline Siatwinda & $23.1(52)$ & nd & $100(95)$ & $100(45)$ \\
\hline Manchanvwa & $52.0(50)$ & $66.7(66)$ & 100 (43) & 100 (60) \\
\hline Control* $^{*}$ & $2.5(120)$ & $3.8(105)$ & $4.9(142)$ & $4.5(112)$ \\
\hline
\end{tabular}

$n=$ number of $A n$. funestus s.l. tested, \% mortality: percentage mortality of $A n$. funestus 24 hours post-exposure; nd = test not done.

${ }^{\star}$ Control tests involved exposure of F1 An. funestus s.l. to untreated papers.

status of An. funestus to bendiocarb in four study sites (Table 3) except in GVDC, where the mortality rates of resistant mosquitoes increased from $40.9 \%$ to $92.0 \%$ (paired sample Student's $t$-test; $t=3.940, \mathrm{df}=4$, and $P=0.029$ ).

Parasite determination. A total of 448 An. funestus mosquitoes were tested for the presence of the circumsporozoite protein of $P$. falciparum from their heads and thoraxes using ELISA. The parasite infection rates of $A$. funestus among the study areas ranged from $0 \%$ in Siatwinda $(n=48)$ and Sikaneka $(n=60)$ to $12.9 \%(95 \% \mathrm{Cl}: 5.0-20.7)$ in Chiyabi area (Table 4). The overall $P$. falciparum parasite prevalence in An. funestus populations was $5.8 \%(95 \% \mathrm{Cl}$ : 3.6-7.9) as summarized in Table 4.

\section{DISCUSSION}

The study demonstrated that $A n$. funestus s.s. and An. rivulorum were the only members of the An. funestus group found in the six study areas of Southern Province after screening 310 samples with PCR. The subspecies of $A n$. funestus group showed evidence of resistance to deltamethrin (a pyrethroid) and bendiocarb (a carbamate) but remained fully susceptible to DDT (an organochlorine) and pirimiphos-methyl (an organophosphate) in Southern Province. Pre-exposure of An. funestus populations to PBO restored the susceptibility of pyrethroid and carbamate resistance observed in five study areas. Nonetheless, carbamate resistance was partially restored in populations of An. funestus from GVDC with $92 \%$ mortality 24 hours post-exposure. The results of the study revealed high variation in the $P$. falciparum infection rates in An. funestus across the six study areas.

Pyrethroid and carbamate cross-resistance reported in southern Zambia mirrors the experience in neighboring Mozambique, ${ }^{46,47}$ Malawi, ${ }^{48,49}$ and Zimbabwe ${ }^{29,50}$ and suggests a regional vector control challenge. Detection of cross-resistance in the malaria vector $A n$. funestus is of major concern and limits insecticide selection choice for resistance management in southern Zambia. The absence of cross-resistance between pyrethroid and DDT in An. funestus across the study areas might suggest that a kdr-type of target-site resistance mechanism has not been selected for by the two insecticides at the time of the study. The complete susceptibility of $A n$. funestus to pirimiphosmethyl and DDT observed in the study areas suggest that the two insecticides are still effective against these species and could be used as alternatives to pyrethroids and carbamates for the effective scale-up of IRS operations in southern Zambia. Nonetheless, DDT cannot be used for IRS operations in areas where An. gambiae s.s. dominate largely because of high levels of resistance documented within this species. ${ }^{24}$ To safeguard the limited number of insecticide classes available for vector control, the WHO Global Plan for Insecticide Resistance Management in Malaria Vectors encourages malaria-endemic countries to develop insecticide resistance management plans based on the local evidence. ${ }^{33,51}$ Adoption of effective insecticide resistance management strategies depends on assessing susceptibility of malaria vector populations to classes of insecticides used for public health. Previous studies conducted in Eastern Province, Zambia, have shown that rotations of non-pyrethroid insecticide classes (such as organophosphate: pirimiphos-methyl) in IRS areas reduced the intensity of resistance of mosquito populations to pyrethroid molecules. ${ }^{23}$

Detection of pyrethroid and carbamate cross-resistance in An. funestus populations in the study areas of Southern Province could be attributed to a number of factors ranging from rapid scale-up of insecticide treated nets (ITNs)/LLINs and IRS, and gene flow to use of pesticide in agriculture. ${ }^{48,52,53}$ Indeed, coverage of pyrethroid-impregnated ITNs increased from $46.9 \%$ in 2006 to $77.8 \%$ in 2015 across the 13 districts of

TABLE 2

Effects of piperonyl butoxide on the mortality of pyrethroid-resistant 2- to 5-day-old F1 Anopheles funestus s.l. mosquitoes in southern Zambia, 2015

\begin{tabular}{|c|c|c|c|c|}
\hline \multirow[b]{2}{*}{ Study site } & \multirow{2}{*}{$\frac{\text { Deltamethrin alone }}{\% \text { Mortality }(n)}$} & \multirow{2}{*}{$\frac{\text { PBO + Deltamethrin }}{\% \text { Mortality }(n)}$} & \multicolumn{2}{|c|}{ Student's $t$-test } \\
\hline & & & $t$-value & $P$-value \\
\hline Buleyamalima & $27.0(100)$ & $100(60)$ & 24.33 & $0.001^{*}$ \\
\hline Gwembe Valley Development Centre & $13.8(100)$ & $100(50)$ & 54.39 & $0.001^{*}$ \\
\hline Chiyabi & $28.0(32)$ & $100(40)$ & 52.28 & $0.012^{*}$ \\
\hline Manchanvwa & $52.0(50)$ & $100(50)$ & 12.00 & 0.053 \\
\hline Sikaneka & $42.3(26)$ & $100(50)$ & 7.667 & 0.083 \\
\hline Siatwinda & $23.1(52)$ & $100(50)$ & 19.98 & $0.032^{*}$ \\
\hline
\end{tabular}

PBO = piperonyl butoxide.

${ }^{\star} P<0.05$, paired Student's $t$-test was used to compare An. funestus mortality with deltamethrin alone and when combined with PBO. 
TABLE 3

Effects of piperonyl butoxide on mortality of carbamate-resistant 2- to 5-day-old F1 Anopheles funestus s.l. mosquitoes in southern Zambia, 2015

\begin{tabular}{|c|c|c|c|c|}
\hline \multirow[b]{2}{*}{ Study site } & \multirow{2}{*}{$\frac{\text { Bendiocarb alone }}{\% \text { Mortality }(n)}$} & \multirow{2}{*}{$\frac{\text { PBO + Bendiocarb }}{\% \text { Mortality }(n)}$} & \multicolumn{2}{|c|}{ Student's $t$-test } \\
\hline & & & $t$-value & $P$-value \\
\hline Buleyamalima & $55.3(94)$ & $100(40)$ & 3.804 & $0.031^{*}$ \\
\hline Gwembe Valley Development Centre & $40.9(93)$ & $92.0(50)$ & 3.940 & $0.029^{\star}$ \\
\hline Chiyabi & $47.0(60)$ & $100(50)$ & 7.341 & $0.018^{\star}$ \\
\hline Manchanvwa & $66.7(66)$ & $100(44)$ & 1.783 & 0.216 \\
\hline Sikaneka & $56.0(50)$ & $100(50)$ & 2.750 & 0.222 \\
\hline
\end{tabular}

${ }^{*} \mathrm{P}<0.05$, paired Student's $t$-test was used to compare An. funestus mortality with bendiocarb alone and when combined with PBO.

Southern Province and might contribute to the pyrethroid resistance profiles being reported in the study areas. Furthermore, review of insecticides used for agriculture (Supplement Table S1) shows that a number of carbamates are used in the study areas for agriculture ${ }^{54,55}$ and could be responsible for selecting carbamate resistance in An. funestus populations. ${ }^{52}$ In addition, the observed carbamate resistance in $A n$. funestus within the study areas could be related to pyrethroid crossresistance mediated by metabolic mechanisms as previously reported from Zimbabwe, Malawi, and South Africa. 50,56,57

Understanding the role of the metabolic resistance mechanism in malaria vectors is critical for the effective implementation of insecticide-based vector interventions. In this study, preexposure of An. funestus populations to PBO restored susceptibility of pyrethroid and carbamate in the study areas, suggesting that P450 monooxygenases play a role in the resistance phenotype. By contrast, the partial recovery of $A n$. funestus susceptibility after PBO pre-exposure to carbamates in a highly mechanized agricultural area of GVDC highlights the possible presence of other metabolic enzyme groups in the study areas besides P450 monooxygenases. The study findings further suggest that PBO-impregnated bednets (PermaNet ${ }^{\circledR}$ 3.0 [Vestergaard Frandsen, Saint Francois, Switzerland] and Olyset Net Plus ${ }^{\circledR}$ [Sumitomo Chemicals, Chuo, Japan]) could counter such resistance mechanisms and aid malaria reduction in Southern Province, Zambia. The role of P450 monooxygenases in causing high pyrethroid resistance in An. funestus and An. gambiae s.l. populations has been previously reported in Zambia, 22,25,30,31 and the information guided the National Malaria Control Programme to select effective resistance management strategies for malaria control and elimination.

Assessing vector competence is usually achieved through finding an infectious stage of the malaria parasite (the sporozoite) in the salivary glands of a mosquito. High $P$. falciparum sporozoite infection rates in An. funestus were recorded in four study areas: Manchanvwa, GVDC, Buleyamalima, and

TABLE 4

Plasmodium falciparum infection rates in Anopheles funestus s.l. in the Southern Province of Zambia, 2015

\begin{tabular}{lccc}
\hline \multicolumn{1}{c}{ Study site } & $\begin{array}{c}\text { Number of } \\
\text { mosquitoes } \\
\text { tested }\end{array}$ & $\begin{array}{c}\text { Number positive } \\
\text { for Plasmodium } \\
\text { falciparum }\end{array}$ & $\begin{array}{c}\text { \% Sporozoite } \\
\text { rate }\end{array}$ \\
\hline Buleyamalima & 90 & 8 & 8.9 \\
Gwembe Valley & 116 & 6 & 5.2 \\
$\quad \begin{array}{l}\text { Development Centre } \\
\text { Chiyabi }\end{array}$ & 70 & 9 & 12.9 \\
Manchanvwa & 64 & 3 & 4.7 \\
Siatwinda & 48 & 0 & 0.0 \\
Sikaneka & 60 & 0 & 0.0 \\
Total & 448 & 26 & 5.8 \\
\hline
\end{tabular}

Chiyabi, whereas no sporozoites were found in mosquitoes from Sikaneka and Siatwinda. The detection of parasite infection rates of $5.8 \%$ in populations of $A n$. funestus from these four study areas of southern Zambia highlight its potential role in malaria transmission and requires an effective integrated vector control program. These study findings agree with previous studies that documented a parasite rate of 10\% in 2013 in An. funestus in Southern Province. ${ }^{25}$ By contrast, the absence of malaria parasites in An. funestus from Sikaneka and Siatwinda could be associated with high spray coverages exceeding $85 \%$ during the 2014 IRS campaigns compared to other study areas. The findings are consistent with previous studies which demonstrated the impact of IRS on reducing sporozoite infection rates of An. funestus in the presence of pyrethroid resistance in Bioko Island ${ }^{58}$ and Mozambique. ${ }^{59}$ However, because of the fact that only 448 samples were analyzed, more studies are required to better assess infectivity rates across the sites. Despite detecting An. rivulorum in the study areas, its epidemiological role in malaria transmission remains unknown in southern Zambia. Nonetheless, previous studies conducted in Tanzania ${ }^{60}$ and Kenya have detected $P$. falciparum in An. rivulorum. ${ }^{61}$

Our study had some limitations. The small sample size of An. funestus mosquitoes used for the determination of WHO susceptibility and resistance bioassays provided information on a limited scale to infer the presence but not the level of resistance in the study areas. Wider scale studies with adequate sample size from not only Southern Province but also from other sites across the country are needed to better inform the insecticide resistance profile of vectors in Zambia. We also had insufficient resources (including a lack of PCR primers) to analyze all specimens to confirm species identities, and so not all samples that were exposed to insecticides underwent PCR to determine the sibling species of the funestus group. Although most appeared to be An. funestus s.s., we cannot confidently associate the resistant phenotypes seen with that species alone. Furthermore, resources were limited to perform molecular genotyping to characterize resistance mechanisms in the populations of $A n$. funestus in the study areas. There is a need for further studies to determine more comprehensively the insecticide resistance mechanisms present in the study areas to guide the technical advisory committee of insecticide resistance management at the national scale.

Detection of pyrethroid and carbamate resistance in An. funestus populations provides a platform for increased insecticide resistance monitoring along Lake Kariba. To prevent the development of metabolic resistant mosquitoes and adequately reduce the underlying vectorial capacity in areas targeted for malaria elimination in southern Zambia, adoption of an effective integrated vector management strategy based 
on local empirical evidence will be needed. An integrated vector management strategy focused on strengthening collaboration ties between its public health and agricultural sectors, encouraging rotational use of IRS insecticides, integrating PBO LLINs and community-based larviciding, house screening, and enhancing insecticide resistance monitoring should be considered. New insecticides for both IRS (clothianidin, chlorfenapyr, and Fludora Fusion) and LLINs (Interceptor G2 and Royal Guard) have been developed in recent years, and they may be valuable additions in extending Zambia's vector control arsenal as the country seeks malaria elimination by 2021 .

Received September 6, 2019. Accepted for publication April 8, 2020.

Published online July 2, 2020.

Note: Supplemental table appears at www.ajtmh.org.

Acknowledgments: We are very grateful for the assistance rendered by community health workers and the Zambian Ministry of Health at the district, provincial, and national levels. We sincerely thank Daniel Bridges for coordinating the molecular analysis of mosquito samples at the National Malaria Elimination Centre in Lusaka. We also thank the Centers for Disease Control and Prevention and Vestergaard for providing the ELISA reagents for sporozoite detection and the synergist PBO for the identification of metabolic resistance mechanisms. We would like to thank Nabie Bayoh for assisting with the statistical analysis. We would also like to thank Larry Slutsker for the timely and valuable comments he provided for this manuscript. Last, we would like to thank Elizabeth Chizema, director of the National Malaria Elimination Centre and of disease surveillance, control, and research at the Zambia Ministry of Health, for her support toward fighting the malaria scourge in Southern Province and all of Zambia.

Financial support: This work was supported by funding from the Bill \& Melinda Gates Foundation through a grant to the PATH Malaria Control and Elimination Partnership in Africa (MACEPA) and the Entomological Surveillance Department in Zambia.

Authors' addresses: Javan Chanda, Kochelani Saili, Mulenga Mwenda, Sandra Chishimba, Conceptor Mulube, Brenda Mambwe, Christopher Lungu, Duncan Earle, and John M. Miller, PATH Malaria Control and Elimination Partnership in Africa (MACEPA), Lusaka, Zambia, E-mails: jchanda@path.org, kochelanisail@gmail.com, mchimfwembe@path.org, schishimba@path.org, cmulube@path.org, bmambwe@path.org, clungu@path.org, dearle@path.org, and jmiller@path.org. Foustina Phiri and Mulakwa Kamuliwo, National Malaria Elimination Centre, Zambia Ministry of Health, Lusaka, Zambia, E-mails: ngawatina@ yahoo.com and mkamuliwo@yahoo.co.uk. Jennifer C. Stevenson, Macha Research Trust, Choma, Zambia, and W. Harry Feinstone Department of Molecular Microbiology and Immunology, Johns Hopkins Bloomberg School of Public Health, Baltimore, MD, E-mail: jennyc.stevenson@macharesearch.org. Adam Bennett, Malaria Elimination Initiative, Global Health Group, University of California San Francisco, San Francisco, CA, E-mail: adam.bennett@ucsf.edu. Thomas P. Eisele and Joseph Keating, Department of Tropical Medicine, Center for Applied Malaria Research and Evaluation, Tulane University School of Public Health and Tropical Medicine, New Orleans, LA, E-mails: teisele@tulane.edu and jkeating@tulane.edu. Richard W. Steketee, President's Malaria Initiative, U.S. Agency for International Development, GH/AA/PMI, Washington, DC, E-mail: rsteketee@path.org. Chadwick H. Sikaala, National Malaria Elimination Centre, Zambia Ministry of Health, Lusaka, Zambia, and SADC Malaria Elimination Eight Secretariat, Windhoek, Namibia, E-mail: chadsikaala@gmail.com.

This is an open-access article distributed under the terms of the Creative Commons Attribution (CC-BY) License, which permits unrestricted use, distribution, and reproduction in any medium, provided the original author and source are credited.

\section{REFERENCES}

1. World Health Organization, 2019. World Malaria Report 2019. Geneva, Switzerland: WHO.
2. National Malaria Elimination Centre, 2019. Midterm Review of the National Malaria Elimination Strategic Plan (2017-2021). Lusaka, Zambia: National Malaria Elimination Programme.

3. Bhatt S et al., 2015. The effect of malaria control on Plasmodium falciparum in Africa between 2000 and 2015. Nature 526: 207-211.

4. Hemingway J, Shretta R, Wells TNC, Bell D, Djimdé AA, Achee N, Qi G, 2016. Tools and strategies for malaria control and elimination: what do we need to achieve a grand convergence in malaria? PLoS Biol 14: e1002380.

5. Hemingway $\mathrm{J}$ et al., 2016. Averting a malaria disaster: will insecticide resistance derail malaria control? Lancet 387: 1785-1788.

6. Bransby-Williams WR, 1979. House catches of adult Anopheles gambiae species B in two areas of Zambia. East Afr Med $J$ 56: 557-561.

7. World Health Organization, 2009. WHO Recommended Insecticides for Indoor Residual Spraying against Malaria Vectors. Geneva, Switzerland: WHO.

8. Zaim M, Aitio A, Nakashima N, 2000. Safety of pyrethroid-treated mosquito nets. Med Vet Entomol 14: 1-5.

9. Camara S, Ahoua Alou LP, Amanan Koffi A, Clegban YCM, Kabran JP, Koffi FM, Koffi K, Pennetier C, 2018. Efficacy of Interceptor $\AA$ G2, a new long-lasting insecticidal net against wild pyrethroid-resistant Anopheles gambiae ss from Côte d'Ivoire: a semi-field trial. Parasite 25: 42.

10. Chanda E, Phiri FN, Chanda J, Baboo KS, 2012. Impact of entomological interventions on malaria vector bionomics in low transmission settings in Zambia. J Public Health Epidemiol 4: 189-196.

11. Hamainza B, Sikaala $\mathrm{CH}$, Moonga HB, Chanda J, Chinula D, Mwenda M, Kamuliwo M, Bennett A, Seyoum A, Killeen GF, 2016. Incremental impact upon malaria transmission of supplementing pyrethroid-impregnated long-lasting insecticidal nets with indoor residual spraying using pyrethroids or the organophosphate, pirimiphos methyl. Malar J 15: 100

12. Protopopoff N, Wright A, West PA, Tigererwa R, Mosha FW, Kisinza W, Kleinschmidt I, Rowland M, 2015. Combination of insecticide treated nets and indoor residual spraying in northern Tanzania provides additional reduction in vector population density and malaria transmission rates compared to insecticide treated nets alone: a randomised control trial. PLoS One 10: e0142671.

13. The malERA Consultative Group on Vector Control, 2011. A research agenda for malaria eradication: vector control. PLOS Med 8: e1000401.

14. Scott TW, Takken W, 2012. Feeding strategies of anthropophilic mosquitoes result in increased risk of pathogen transmission. Trends Parasitol 28: 114-121.

15. Akogbéto MC, Djouaka RF, Kindé-gazard DA, 2006. Screening of pesticide residues in soil and water samples from agricultural settings. Malar J 9: 1-9.

16. Hemingway $\mathrm{J}$, Ranson $\mathrm{H}, 2000$. Insecticide resistance in insect vectors of human disease. Annu Rev Entomol 45: 371-391.

17. Roberts DR, Andre RG, 1994. Insecticide resistance issues in vector-borne disease control. Am J Trop Med Hyg 50: 21-34.

18. Brogdon WG, McAllister JC, 1998. Insecticide resistance and vector control. Emerg Infect Dis 4: 605.

19. Wood OR, Hanrahan S, Coetzee M, Koekemoer LL, Brooke BD, 2010. Cuticle thickening associated with pyrethroid resistance in the major malaria vector Anopheles funestus. Parasit Vectors 3: 67.

20. Sokhna C, Ndiath MO, Rogier C, 2013. The changes in mosquito vector behaviour and the emerging resistance to insecticides will challenge the decline of malaria. Clin Microbiol Infect 19: 902-907.

21. Zalucki MP, Furlong MJ, 2017. Behavior as a mechanism of insecticide resistance: evaluation of the evidence. Curr Opin Insect Sci 21: 19-25.

22. Chanda $E$ et al., 2011. Insecticide resistance and the future of malaria control in Zambia. PLoS One 6: e24336.

23. Chanda $\mathrm{E}$ et al., 2016. An operational framework for insecticide resistance management planning. Emerg Infect Dis 22: 773-779.

24. Ministry of Health, Zambia, 2014. Zambia Insecticide Resistance Management Plan. Lusaka, Zambia: National Malaria Control Programme, 1-27. 
25. Thomsen EK et al., 2014. Underpinning sustainable vector control through informed insecticide resistance management. PLOS One 9: e99822.

26. Ranson $\mathrm{H}$, Lissenden N, 2016. Insecticide resistance in African Anopheles mosquitoes: a worsening situation that needs urgent action to maintain malaria control. Trends Parasitol 32: 187-196.

27. Eisele TP et al., 2016. Short-term impact of mass drug administration with dihydroartemisinin plus piperaquine on malaria in Southern Province Zambia: a cluster-randomized controlled trial. J Infect Dis 214: 1831-1839.

28. Ministry of Health, Zambia, 2012. Zambia National Malaria Indicator Survey 2012. Lusaka, Zambia: National Malaria Control Programme.

29. Choi KS et al., 2014. Insecticide resistance and role in malaria transmission of Anopheles funestus populations from Zambia and Zimbabwe. Parasit Vectors 7: 464.

30. Riveron JM, Ibrahim SS, Chanda E, Mzilahowa T, Cuamba N, Irving $\mathrm{H}$, Barnes KG, Ndula M, Wondji CS, 2014. The highly polymorphic CYP6M7 cytochrome P450 gene partners with the directionally selected CYP6P9a and CYP6P9b genes to expand the pyrethroid resistance front in the malaria vector Anopheles funestus in Africa. BMC Genomics 15: 817.

31. Chanda E, Chanda J, Kandyata A, Phiri FN, Muzia L, Haque U, Baboo KS, 2013. Efficacy of ACTELLIC $300 \mathrm{CS}$, pirimiphos methyl, for indoor residual spraying in areas of high vector resistance to pyrethroids and carbamates in Zambia. $J$ Med Entomol 50: 1275-1281.

32. Wondji CS, Irving H, Morgan J, Lobo NF, Collins FH, Hunt RH, Coetzee M, Hemingway J, Ranson H, 2009. Two duplicated P450 genes are associated with pyrethroid resistance in Anopheles funestus, a major malaria vector. Genome Res 19: 452-459.

33. World Health Organization, 2012. Global Plan for Insecticide Resistance Management in Malaria Vectors. Geneva, Switzerland: WHO.

34. Larsen DA et al., 2015. Malaria surveillance in low-transmission areas of Zambia using reactive case detection. Malar J 14: 465.

35. Kent RJ, Thuma PE, Mharakurwa S, Norris DE, 2007. Seasonality, blood feeding behavior, and transmission of Plasmodium falciparum by Anopheles arabiensis after an extended drought in Southern Zambia. Am J Trop Med Hyg 76: 267-274.

36. Chimumbwa JM, 2003. The Epidemiology of Malaria in Zambia. Pretoria, South Africa: University of Pretoria.

37. Morgan JC, Irving H, Okedi LM, Steven A, Wondji CS, 2010. Pyrethroid resistance in an Anopheles funestus population from Uganda. PLoS One 5: 1-8.

38. World Health Organization, 2013. Test Procedures for Insecticide Resistance Monitoring in Malaria Vector Mosquitoes. Geneva, Switzerland: WHO.

39. Brogdon W, Chan TK, 2011. Guideline for Evaluating Insecticide Resistance in Vectors Using the CDC Bottle Bioassay. Atlanta, GA: Centers for Disease Control and Prevention.

40. Kloke RG, Nhamahanga E, Hunt RH, Coetzee M, 2011. Vectorial status and insecticide resistance of Anopheles funestus from a sugar estate in Southern Mozambique. Parasit Vectors 4: 16.

41. Wirtz RA, Zavala F, Charoenvit Y, Campbell GH, Burkot TR, Schneider I, Esser KM, Beaudoin RL, Andre RG, 1987. Comparative testing of monoclonal antibodies against Plasmodium falciparum sporozoites for ELISA development. Bull World Health Organ 65: 39.

42. Gillies MT, De Meillon B, 1968. The Anophelinae of Africa south of the sahara (Ethiopian zoogeographical region). Publ S Afr Inst Med Res 54: 1-343.

43. Gillies MT, Coetzee M, 1987. A supplement to the anophelinae of Africa south of the sahara. Publ Afr Inst Med Res 55: 1-143.
44. Collins FH, Mendez MA, Rasmussen MO, Mehaffey PC, Besansky NJ, Finnerty V, 1987. A ribosomal RNA gene probe differentiates member species of the Anopheles gambiae complex. Am J Trop Med Hyg 37: 37-41.

45. Koekemoer LL, Kamau L, Hunt RH, Coetzee M, 2002. A cocktail polymerase chain reaction assay to identify members of the Anopheles funestus (Diptera: Culicidae) group. Am J Trop Med Hyg 66: 804-811.

46. Casimiro S, Coleman M, Mohloai P, Hemingway J, Sharp B, 2006. Insecticide resistance in Anopheles funestus (Diptera: culicidae) from Mozambique. J Med Entomol 43: 267-275.

47. Cuamba N, Morgan JC, Irving H, Steven A, Wondji CS, 2010. High level of pyrethroid resistance in an Anopheles funestus population of the Chokwe district in Mozambique. PLoS One 5: e11010.

48. Hunt RH, Edwardes M, Coetzee M, 2010. Pyrethroid resistance in Southern African Anopheles funestus extends to Likoma Island in Lake Malawi. Parasit Vectors 3: 122.

49. Mzilahowa T et al., 2016. Increasing insecticide resistance in Anopheles funestus and Anopheles arabiensis in Malawi, 2011-2015. Malar J 15: 563.

50. Sande S, Zimba M, Chinwada P, Masendu HT, Mazando S, Makuwaza A, 2015. The emergence of insecticide resistance in the major malaria vector Anopheles funestus (Diptera: Culicidae) from sentinel sites in Mutare and Mutasa districts, Zimbabwe. Malar J 14: 466.

51. Mnzava AP, Knox T, Temu EA, Trett A, 2015. Implementation of the global plan for insecticide resistance management in malaria vectors: progress, challenges and the way forward. Malar J 14: 173

52. Yadouleton $A$ et al., 2011. Cotton pest management practices and the selection of pyrethroid resistance in Anopheles gambiae population in northern Benin. Parasit Vectors 4: 1-11.

53. Implications of Insecticide Resistance Consortium, 2018. Implications of insecticide resistance for malaria vector control with long-lasting insecticidal nets: trends in pyrethroid resistance during a WHO-coordinated multi-country prospective study. Parasit Vectors 11: 550

54. Ministry of Finance, 2013. E4153 Ministry of Finance Strategic Programme for Climate Resilence in Zambia. Lusaka, Zambia: Ministry of Agriculture and Livestock.

55. Ministry of Agriculture and Livestock, 2011. Livestock Development and Animal Volume III (without ESMF and RPF). III. Lusaka, Zambia: Ministry of Agriculture and Livestock.

56. Riveron JM et al., 2015. Rise of multiple insecticide resistance in Anopheles funestus in Malawi: a major concern for malaria vector control. Malar J 14: 344

57. Hargreaves K, Koekemoer LL, Brooke BD, Hunt RH, Mthembu J, Coetzee M, 2000. Anopheles funestus resistant to pyrethroid insecticides in South Africa. Med Vet Entomol 14: 181-189.

58. Sharp BL, Ridl FC, Govender D, Kuklinski J, Kleinschmidt I, 2007. Malaria vector control by indoor residual insecticide spraying on the tropical island of Bioko, Equatorial Guinea. Malar J 6: 52.

59. Abilio AP et al., 2011. The emergence of insecticide resistance in central Mozambique and potential threat to the successful indoor residual spraying malaria control programme. Malar J 10: 110.

60. Wilkes TJ, Matola YG, Charlwood JD, 1996. Anopheles rivulorum, a vector of human malaria in Africa. Med Vet Entomol 10: 108-110.

61. Kawada H, Dida GO, Sonye G, Njenga SM, Mwandawiro C, Minakawa N, 2012. Reconsideration of Anopheles rivulorum as a vector of Plasmodium falciparum in western Kenya: some evidence from biting time, blood preference, sporozoite positive rate, and pyrethroid resistance. Parasit Vectors 5: 230. 